\title{
Using the Methodology of Systematic Review of Reviews for Evidence-Based Medicine \\ Edris Hasanpoor ${ }^{1}$, Jamal Hallajzadeh ${ }^{2}$, Yibeltal Siraneh ${ }^{3}$, Ebrahim Hasanzadeh $^{4}$, Elaheh Haghgoshayie ${ }^{1 *}$
}

\author{
OPEN ACCESS \\ Citation: Edris Hasanpoor, Jamal \\ Hallajzadeh, Yibeltal Siraneh, Ebrahim \\ Hasanzadeh, Elaheh Haghgoshayie. \\ Using the Methodology of Systematic \\ Review of Reviews for Evidence-Based \\ Medicine. Ethiop J Health Sci. 2019; \\ 29(6):775.doi:http://dx.doi.org/10.4314/ej \\ hs.v29 i6.15 \\ Received: August 06, 2019 \\ Accepted: August 23, 2019 \\ Published: November 1, 2019 \\ Copyright: (C2019 Hasanpoor E, et al. \\ This is an open access article distributed \\ under the terms of the Creative Commons \\ Attribution License, which permits \\ unrestricted use, distribution, and \\ reproduction in any medium, provided the \\ original author and source are credited. \\ Funding: Nil \\ Competing Interests: The authors \\ declare that this manuscript was approved \\ by all authors in its form and that no \\ competing interest exists. \\ Affiliation and Correspondence: \\ ${ }^{1}$ Department of Healthcare \\ Management, Research Center for \\ Evidence-Based Health Management, \\ Maragheh University of Medical \\ Sciences, Maragheh, Iran \\ ${ }^{2}$ Department of Clinical Biochemistry, \\ Research Center for Evidence-Based \\ Health Management, Maragheh \\ University of Medical Sciences, \\ Maragheh, Iran \\ ${ }^{3}$ Department of Health Economics, \\ Management and Policy, Faculty of \\ Public Health, Institute of Health, \\ Jimma University, Jimma, Ethiopia \\ ${ }^{4}$ Student Research Committee, \\ Department of Health Services \\ Management, Iran University of \\ Medical Sciences, Tehran, Iran \\ *Email: ezd_ehm2010@yahoo.com
}

\section{Introduction}

Evidence-based medicine miracle has embraced all the sciences (15). This movement improved medical practice using the critical thinking (1, 6-9). In the meantime, systematic reviews are one of the best available evidence by apprising preliminary studies. Systematic reviews and meta-analyses have become increasingly important in health care. In addition, these studies are increasing globally in different healthcare settings (10-13). It is estimated that around 22 new systematic reviews are published every day. The evidence suggest that almost 50 systematic reviews are published every day (14). The purpose of this study was to introduce the type, necessities, and applications of reviews of systematic reviews for evidence-based medicine.

With the increase in the number of systematic reviews, a fundamental next step to provide decision-makers in healthcare with the evidence they require has been the conduct of reviews of existing systematic reviews and evidence brief. The reviews of systematic reviews are referred to by several different terms in the scientific evidence, including umbrella reviews, overviews of reviews, metareview, review of reviews, a summary of systematic reviews and also a synthesis of reviews (15). The reviews of systematic reviews are a relatively new method of evidence synthesis. Each reviews of systematic reviews focuses on a broad condition or problem for which there are two or more potential interventions and highlights reviews that address these potential interventions and their results $(15,16)$.

Conduct of a meta-review offers the possibility to address a broad area of issues related to the phenomena of interest and is ideal to present a wide scope of the evidence related to a specific question $(15,17)$. Systematic reviews are essential to evidence-based decision-making and provide the highest level of evidence to clinicians and policy-makers (18-21). Nowadays more and more number of review studies is increasingly expanding and are becoming part of the routine and basic research in healthcare sciences (22). On the other hand, clinicians and policy-makers are faced with a large number of review studies in a particular domain of work $(2,3,6)$. So, when collecting and extracting outcomes from several reviews, the reviews of systematic reviews is needed. In 
literature, many topics have multiple systematic reviews with varying degrees of quality and scope. Thus, reviews of systematic reviews is needed to finalize into a single document where the results of multiple reviews can be compared, thus making it easier for the decision-maker $(16,23)$.

\section{Methodology of Systematic Review of Reviews}

The meta-review or reviews of systematic reviews is important for several reasons $(10,24,25)$ : (1) it allows the results of reviews relevant to a review question to be compared and contrasted; (2) It allows ready assessment of whether review authors addressing similar review questions independently observe similar findings; (3) it can play a role in signposting the reader to evidence, summarising existing research or highlighting the absence of evidence; (4) It could be a useful source for decision-makers in developing clinical practice guidelines, decision support systems, and drug formularies; (5) the reviews of systematic reviews contain a clearly stated objective designed to answer at least one research question; and (6) It was judged to be the most appropriate method to address this complex area of as there is a vast literature which is highly heterogeneous. Conducting reviews of systematic reviews enables the results of individual reviews to be brought together, compared and contrasted, with the aim of providing a single comprehensive overview, which can serve as a simple introduction to the challenges of achieving change and implementing complex interventions in healthcare services for managers, clinicians or decision- makers $(5,24$, $25)$. According to evidence, there is different type of reviews of systematic reviews. Common applications for systematic review of reviews based on the type of included reviews in reviews of systematic reviews are shown in Table 1.

Table 1. Applications for different systematic review of reviews.

\begin{tabular}{|c|c|c|}
\hline $\mathbf{0}$ & $\begin{array}{l}\text { Type of systematic review } \\
\text { of reviews }\end{array}$ & Applications \\
\hline 1 & $\begin{array}{l}\text { Systematic review of } \\
\text { intervention reviews }\end{array}$ & $\begin{array}{l}\text { To summarise evidence from more than one systematic review of different } \\
\text { interventions for the same condition or problem. } \\
\text { To summarise evidence from more than one systematic review of the same } \\
\text { intervention for the same condition or problem where different outcome } \\
\text { To summarise evidence from more than one systematic review of the same } \\
\text { intervention for the same condition or problem where different outcomes. } \\
\text { To summarise evidence from more than one systematic review of the same } \\
\text { intervention for different conditions, problems or populations. } \\
\text { To summarise evidence about adverse effects of an intervention from } \\
\text { more than one systematic review of use of the intervention for one or more } \\
\text { conditions. }\end{array}$ \\
\hline 2 & $\begin{array}{l}\text { Systematic re } \\
\text { prognosis/pre } \\
\text { review }\end{array}$ & $\begin{array}{l}\text { To summarise evidence about prognosis/ prevalence from more than one } \\
\text { systematic review. }\end{array}$ \\
\hline 3 & $\begin{array}{l}\text { Systematic review of } \\
\text { diagnostic test accuracy } \\
\text { reviews }\end{array}$ & $\begin{array}{l}\text { To summarise evidence from more than one systematic review of } \\
\text { diagnostic test accuracy assessing the same medical test to address the } \\
\text { same condition or problem. }\end{array}$ \\
\hline 4 & $\begin{array}{l}\text { Systematic review of risk } \\
\text { factors reviews }\end{array}$ & $\begin{array}{l}\text { These overviews incorporate disease etiology or risk factors when the } \\
\text { risks of interest may not directly relate to prognostic variables or risk } \\
\text { prediction models. }\end{array}$ \\
\hline 5 & $\begin{array}{l}\text { Systeme } \\
\text { descrip }\end{array}$ & $\begin{array}{l}\text { To summarise evidence from more than one systematic review of } \\
\text { descriptive reviews to address the descriptive results of review studies. }\end{array}$ \\
\hline 6 & $\begin{array}{l}\text { Systematic review of } \\
\text { qualitative reviews }\end{array}$ & $\begin{array}{l}\text { To summarise systematic review evidence relating to qualitative views or } \\
\text { experiences. There is clear guidance available on the good conduct of an } \\
\text { overview of qualitative syntheses. }\end{array}$ \\
\hline
\end{tabular}

DOI: http://dx.doi.org/10.4314/ejhs.v29i6.15 
A meta-review's most important feature is that this type of evidence synthesis only assumes for inclusion the highest level of evidence, namely other systematic reviews and meta-analyses (13, $15,23)$. Specifically, review of systematic reviews refers to review compiling evidence from multiple reviews into one accessible and usable document. Focuses on broad condition or problem for which there are competing interventions and highlights reviews that address these interventions and their results. However, as systematic reviews become more numerous, there is the potential for greater use of such overarching reviews as a mechanism for aggregating findings from several reviews that address specific questions $(15,23)$. With the everincreasing number of systematic reviews published daily, reviews of systematic reviews have a clear role in evidence-based healthcare and decision-making in health care services $(2,6,26$, 27). This era is known as age of the evidencebased everything. Healthcare decision-makers can create a conducive context and provide the big opportunities to improve peer-to-peer evidence and knowledge sharing together. The reviews of systematic reviews have the potential to be a useful tool for the translation of health evidence and decision-making. In addition, reviews of systematic reviews are sources of evidence for conducting the policy brief and for designing the decision support systems. A systematic review of reviews allows the creation of a summary of reviews in a single document. These may be preferred by healthcare decision-makers because they synthesize all systematic reviews evidence in one single document. In future study, we identify the items based on the best available evidence for reporting the systematic review of reviews.

\section{REFERENCES}

1. Hasanpoor E, Bahadori M, Yaghoubi M, Haghgoshayie E, Mahboub-Ahari A. Evidence-based management as a basis for evidence-based medical consultation. $B M J$ evidence-based medicine. 2018:bmjebm-2018111104.

2. Hasanpoor E, Hajebrahimi S, Janati A, Abedini Z, Haghgoshayie E. Barriers, facilitators, process and sources of evidence for evidence-based management among health care managers: a qualitative systematic review. Ethiopian journal of health sciences. 2018;28(5):665.

3. Hasanpoor E, Janati A, Arab-Zozani M, Haghgoshayie E. Using the evidence-based medicine and evidence-based management to minimise overuse and maximise quality in healthcare: a hybrid perspective. $B M J$ evidence-based medicine. 2018:bmjebm-2018110957.

4. Hasanpoor E, Belete YS, Janati A, Hajebrahimi S, Haghgoshayie E. The Use of Evidence-Based Management in Nursing Management. Africa Journal of Nursing and Midwifery. 2019:9;21(1):16.

5. Langell JT. Evidence-based medicine: A datadriven approach to lean healthcare operations. International Journal of Healthcare Management. 2019:1-4.

6. Janati A, Hasanpoor E, Hajebrahimi S, Sadeghi-Bazargani H. Health care managers' perspectives on the sources of evidence in evidence-based hospital management: A qualitative study in Iran. Ethiopian journal of health sciences. 2017;27(6):659-68.

7. Janati A, Hasanpoor E, Hajebrahimi S, Sadeghi-Bazargani H. Evidence-based management-healthcare manager viewpoints. International journal of health care quality assurance. 2018;31(5):436-48.

8. Janati A, Hasanpoor E, Hajebrahimi S, Sadeghi-Bazargani H, Khezri A. An evidencebased framework for evidence-based management in healthcare organizations: a delphi study. Ethiopian journal of health sciences. 2018;28(3):305-14.

9. Hasanpoor E, Belete YS, HSM PF, Janati A, Hajebrahimi S, Haghgoshayie E. Nursing Managers' Perspectives on the Facilitators and Barriers to Implementation of Evidence-Based Management. Worldviews on Evidence-Based Nursing. 2019;1:8.

10. Moher D, Liberati A, Tetzlaff J, Altman DG, Group P. Preferred reporting items for systematic reviews and meta-analyses: the

DOI: http://dx.doi.org/10.4314/ejhs.v29i6.15 
PRISMA statement. PLoS medicine. 2009;6(7):e1000097.

11. Moher D, Liberati A, Tetzlaff J, Altman DG, Group P. Preferred reporting items for systematic reviews and meta-analyses: the PRISMA statement,2010;336.

12. Moher D, Shamseer L, Clarke M, Ghersi D, Liberati A, Petticrew M, et al. Preferred reporting items for systematic review and meta-analysis protocols (PRISMA-P) 2015 statement. Systematic reviews. 2015;4(1):1.

13. Khan KS, Ball E, Fox CE, Meads C. Systematic reviews to evaluate causation: an overview of methods and application. BMJ evidence-based medicine. 2012;17(5):137-41.

14. Hunt H, Pollock A, Campbell P, Estcourt L, Brunton G. An introduction to overviews of reviews: planning a relevant research question and objective for an overview. Systematic reviews. 2018;7(1):39.

15. Aromataris E, Fernandez R, Godfrey CM, Holly C, Khalil H, Tungpunkom P. Summarizing systematic reviews: methodological development, conduct and reporting of an umbrella review approach. International journal of evidence-based healthcare. 2015;13(3):132-40.

16. Grant MJ, Booth A. A typology of reviews: an analysis of 14 review types and associated methodologies. Health Information \& Libraries Journal. 2009;26(2):91-108.

17. Aromataris E, Fernandez RS, Godfrey C, Holly C, Khalil H, Tungpunkom P. Methodology for JBI umbrella reviews. 2014.

18. Bastian H, Glasziou P, Chalmers I. Seventyfive trials and eleven systematic reviews a day: how will we ever keep up? PLoS medicine. 2010;7(9):e1000326.

19. Pieper D, Mathes T. Survey of instructions for authors on how to report an update of a systematic review: guidance is needed. $B M J$ evidence-based medicine. 2017:ebmed-2016110609.

20. Perera R, Heneghan C. Interpreting metaanalysis in systematic reviews. BMJ evidencebased medicine. 2008;13(3):67-9.

21. Guo R, Berkshire SD, Fulton LV, Hermanson PM. Predicting intention to use evidencebased management among US healthcare administrators: Application of the theory of planned behavior and structural equation modeling. International Journal of Healthcare Management. 2019;12(1):25-32.

22. Mulrow CD. Systematic reviews: rationale for systematic reviews. Bmj. 1994;309(6954):5979.

23. Samnani SS, Vaska M, Ahmed S, Turin TC. Review typology: The basic types of reviews for synthesizing evidence for the purpose of knowledge translation. Journal of the College of Physicians and Surgeons Pakistan. 2017;27(10):635-41.

24. Smith V, Devane D, Begley CM, Clarke M. Methodology in conducting a systematic review of systematic reviews of healthcare interventions. $B M C$ medical research methodology. 2011;11(1):15.

25. Lau R, Stevenson F, Ong BN, Dziedzic K, Treweek S, Eldridge $\mathrm{S}$, et al. Achieving change in primary care - causes of the evidence to practice gap: systematic reviews of reviews. Implementation Science. 2015;11(1):40.

26. Arab-Zozani M, Pezeshki MZ, KhodayariZarnaq R, Janati A. Medical overuse in the Iranian healthcare system: a systematic review protocol. BMJ open. 2018;8(4):e020355.

27. Jefferson T, Doshi P, Boutron I, Golder S, Heneghan $\mathrm{C}$, Hodkinson $\mathrm{A}$, et al. When to include clinical study reports and regulatory documents in systematic reviews. $B M J$ evidence-based medicine. 2018;23(6):210-7.

DOI: http://dx.doi.org/10.4314/ejhs.v29i6.15 